\title{
Histone demethylase KDM5A enhances cell proliferation, induces EMT in lung adenocarcinoma cells, and have a strong causal association with paclitaxel resistance
}

\author{
Lidong $\mathrm{Xu}^{1}$, Hong $\mathrm{Wu}^{2}$ and $\mathrm{Xun} \mathrm{Hu}^{1} \square$ \\ 'Department of Thoracic Surgery, Jiaxing Second Hospital, Jiaxing-Zhejiang, China, 314000; 2Department of Respiratory Medicine, Jiaxing Sec- \\ ond Hospital, Jiaxing-Zhejiang, China, 314000
}

Recent reports suggest that histone demethylase KDM5A emerges as a new player in the development of drug resistance and thus increases the challenges of chemotherapy. Here, we explore the role of KDM5A in cell proliferation, epithelial-mesenchymal transition (EMT)and its causal association with paclitaxel resistance in lung adenocarcinoma. Paclitaxel-resistant lung adenocarcinoma PTX-Calu-3 cells showed significantly higher IC50 value $(7 \pm 0.176 \mu \mathrm{M})$ upon paclitaxel treatment than lung adenocarcinoma SK-LI-1 (3.6 $\pm 0.005 \mathrm{nM})$, Calu-3 (4.3 $\pm 0.015 \mathrm{nM})$, and A549 $(4.5 \pm 0.106 \mathrm{nM})$ cells. We found that expression of KDM5A and P-glycoprotein (P-gp), which plays a critical role in the development of paclitaxel resistance, were significantly higher in PTXCalu-3 cells compared to SK-LI-1, Calu-3, and A549 cells.. We observed a significant increase in the expression of mesenchymal markers $\mathrm{N}$-cadherin and vimentin, and a concomitant decrease in expression of E-cadherin and a-catenin in PTX-Calu-3 compared to SK-LI-1, Calu-3, and A549 lung cancer cell lines. Transwell Boyden chamber and wound healing assays further demonstrated that a significantly higher number of PTX-Calu-3 cells were invasive and motile compared to SK-LI-1, Calu-3, and A549 cells, thus supporting the role of KDM5A in metastasisassociated processes. Additionally, a significantly higher expression of KDM5A was observed in lung adenocarcinoma patients' samples compared with adjacent normal tissues as well as in PTX-Calu-3 cells compared toSK-LI-1, Calu-3, and A549 cells, as shown both with histochemistry and real time-polymerase chain reaction (RT-PCR). In summary, these results suggest that KDM5A plays a key role in lung adenocarcinoma by promoting proliferation, EMT, and drug resistance to paclitaxel treatment.

Key words: Lung adenocarcinoma; EMT; KDM5A; proliferation; paclitaxel

Received: 25 July, 2020; revised: 10 November, 2020; accepted: 05 January, 2021; available on-line: 16 July, 2021

『e-mail: hxzcf1976@126.com

Acknowledgments of Financial Support: We would like to thank the Department of Thoracic Surgery and Department of Respiratory Medicine, Jiaxing Second Hospital, Jiaxing, Zhejiang, China, 314000 . The study was financially supported by Jiaxing Science and Technology Plan Project. NO: 2018AY32001

Abbreviations: KDM5A, Lysine-specific demethylase 5A; EMT, Epithelial-mesenchymal transition; MTT, 3-(4,5-dimethylthiazol-2-y1)-2, 5-diphenyltetrazolium bromide; PTX, Paclitaxel; P-gp, P-glycoprotein; RT-PCR, Real time-polymerase chain reaction; DMEM, Dulbecco's modified Eagle's medium; FBS, fetal bovine serum; PI, propidium iodide; PMSF, phenylmethylsulfonyl fluoride; DTT, dithiothreitol; DMSO, dimethyl sulfoxide; HRP, horseradish peroxidase; HEPES, 4-(2-hydroxyethyl)-1-piperazineethanesulfonic acid; $\mathrm{KCl}$, potassium chloride; SDS-PAGE, sodium dodecyl sulphate-polyacrylamide gel electrophoresis; PVDF, polyvinylidene fluoride; PBS, phosphate buffer saline; TBST, tris-buffered saline Tween-20

\section{INTRODUCTION}

Over the past few decades, lung cancer has become the most common cancer in the world, with the highest mortality and morbidity rates (de Groot $e t$ al., 2018). Lung cancer is associated with approximately, 2.1 million new cases and around 1.8 million deaths annually, constituting the highest proportion of new cancer cases $(11.6 \%)$ and cancer mortality (18.4\%) in both men and women around the world (Bray et al., 2018). Despite advances in lung cancer diagnosis and treatment, the prognosis is poor with less than $16 \%$ of five-year survival (Roointan et al., 2019). Treatment for lung adenocarcinoma includes surgery, chemotherapy, and radiotherapy (Dinglin et al., 2013). The only treatment of choice in advanced stages of lung adenocarcinoma is chemotherapy (Gandhi et al., 2018). Among the currently used chemotherapeutics, Taxol-derived paclitaxel is a commonly used and effective drug for lung adenocarcinoma (Li et al., 2014; Huang et al., 2017). Initially, paclitaxel was remarkably effective in guiding cancer cells into programmed cell death (Shen et al., 2015). However, due to the heterogeneity of lung adenocarcinoma, a significant population of patients develop resistance to paclitaxel, as part of the development of multidrug resistance (Zahreddine \& Borden, 2013). Targeted therapies such as epidermal-growth-factor receptor (EGFR) (erlotinib or afatinib) or anaplastic lymphoma kinase (ALK) (alectinib or crizotinib) inhibitors significantly improve lung cancer therapy. (Minguet et al., 2016). On the other hand, acquired resistance against targeted tyrosine kinase inhibitors (TKIs), by the activation of alternative pathways such as BRAF, MET or HER-2, is the prominent reason for drug resistance in lung cancer (Nagano et al., 2018). This opens a new opportunity for a novel combination of targeted therapy with the immunotherapeutic agents. Recent reports showed that targeted therapy with EGFR or ALK inhibitors in combination with immunotherapy agents (nivolumab, pembrolizumab, or atezolizumab) resulted in significant improvement in lung cancer treatment and increased overall survival time (Alsaab et al., 2017). Although the combination therapy showed promising results even at the advanced stages of lung cancer, the molecular histology of the tumor tissues and the optimal modes of combining targeted therapy with immunotherapy at low toxicity are still under investigated (Kim et al., 2018). Recent reports suggest that multidrug resistance has a strong causal association with EMT and drives cells towards metastasis (Brabletz et al., 2018). EMT is an important biological process that plays a critical role in driving localized malignant cells towards 
more aggressive metastatic cells. The striking feature of EMT cells is morphological modification from a variety of polarized epithelial shapes into mesenchymal spherical shape (Brabletz et al., 2018). The molecular hallmarks of EMT cells are loss of E-cadherin and $\alpha$-catenin, and gain of $\mathrm{N}$-cadherin and vimentin expression (Serrano-Gomez et al., 2016).

Histone modifications play an important role in the regulation of cellular processes during development (Cedar \& Bergman 2009). KDM5A is a H3K4 demethylase, meaning that it catalyzes demethylation of lysine $4(\mathrm{~K} 4)$ in histone $\mathrm{H} 3$, thus regulating many biological processes (Kooistra \& Helin, 2012). Previous studies showed aberrant expression of KDM5A in retinoblastoma and its binding to tumor suppressor retinoblastoma-binding protein ( $\mathrm{Hu}$ et al., 2018). Mutation in its homolog gene in Drosophila influenced cellular kinetics and differentiation (Kolybaba \& Classen, 2014). Apart from KDM5A role in differentiation during development, recent studies revealed that its expression is significantly elevated in several malignancies, including breast, gastric, ovarian, and acute myeloid cancer (Feng et al., 2017; Shokri et al., 2018; Oser et al., 2019). These studies confirmed that KDM5A can act as an oncogenic driver in various malignancies (Hou et al., 2012).

Although in the recent past, $\mathrm{CaO}$ and others (Cao et al., 2014) have shown that KDM5A plays a critical role in breast cancer metastasis and spreads metastatic cells to the lung, the role of KDM5A in lung adenocarcinoma is not yet evaluated. In the current study, we have found thatKDM5A expression in paclitaxelresistant lung adenocarcinoma cell lines is particularly high and acts as a growth kinetics driver with in cells after exposure to paclitaxel. Further, we found that high expression of KDMA5 stimulates EMT, motility, invasion, and metastasis in drug-resistant lung adenocarcinoma cells. Additionally, we observed high expression of KDM5A in tissue samples from the patients who were on paclitaxel chemotherapy, which suggests a casual association between KDM5A and paclitaxel-resistance in lung adenocarcinoma. Together these results present that KDM5A could be a novel drug target in paclitaxel-mediated drug resistance in lung adenocarcinoma.

\section{MATERIALS AND METHODS}

\section{Lung adenocarcinoma tissue and cell lines}

All the tissue samples of lung adenocarcinoma were approved by the Ethics committee of China. A total of 87 pairs of lung adenocarcinoma and the respective adjacent normal tissue samples were collected from the patients with a complete diagnosed history of lung adenocarcinoma.

Human lung adenocarcinoma cell lines SK-LI-1, Calu3, A549, and MRC-5 (transformed lung fibroblasts) were procured from American Type Culture Collection. Paclitaxel resistant Calu-3 (PTX-Calu-3) cell line was obtained from Wuhan University, China. The cell lines were maintained in Dulbecco's modified Eagle's medium (DMEM) (\#A4192101), supplemented with 1\% penicillin-streptomycin (\#15140130), and 10\% fetal bovine serum (FBS) (\#16000044), in a 5\% $\mathrm{CO}_{2}$ incubator at $37^{\circ} \mathrm{C}$. Paclitaxel-resistant Calu-3 cells were maintained in media containing additional $3 \mathrm{nM}$ paclitaxel.

\section{Chemicals, reagents, and antibodies}

All the chemicals, namely: Bradford reagent, 3-(4, 5-dimethylthiazol-2-y1)-2,5-diphenyltetrazolium bromide (MTT) (\#M5655), dimethyl sulphoxide (DMSO) (\#C6164), dithiothreitol (DT'T) (\#10197777001), propidium iodide (PI) (\#P4170), protease cocktail inhibitor (\#P8340), and phenylmethylsulphonyl (PMSF) (\#78830) were procured from Sigma-Aldrich. The primary antibodies; KDM5A (\#MA5-34682, 1:1000 dilution) and P-gp (\#MA1-26528 1:1000 dilution were obtained from Thermo Fisher Scientific. All other antibodies including E-cadherin (\#14472S, 1:2000 dilution), $\alpha$-catenin (\#2131S, 1:1000 dilution), Vimentin (\#5741S, 1:1000 dilution), N-cadherin (\#13116S, 1:1000 dilution), and $\beta$-actin (\#3700S, 1:10000 dilution), and secondary: anti-rabbit (\#7074S, 1:2000 dilution) and anti-mouse (\#93702S, 1:2000 dilution) IgG coupled with horseradish peroxidase were purchased from Cell Signaling Technology.

\section{Transient Transfection}

After obtaining more than 90\% confluency, lung adenocarcinoma cells were transiently transfected with Lipofectamine 2000 (\#11668019, Invitrogen), and either KDM5A plasmid (pBabe-puro/HA-FLAG-RBP2 H483A, obtained from Addgene) or KDM5A siRNA, to either overexpress or knockdown KDM5A, respectively. After $48 \mathrm{~h}$ of transfection the cells were processed for various experiments to evaluate the respective effects. The sequence of KDM5A siRNA was designed and synthesized by Gene Pharma. The target sequences are as follows: \#1 5'-AAGAGCUACAACAGGCUCGGU-3' (sense), siRNA \#2 5'AAGUCCUCUAGUAGUCUUGAA-3' (sense), and siRNA control 5'-UUCUCCGAACGUGUCACGUT'T-3’ (sense).

\section{RNA extraction and real-time quantitative polymerase chain reaction (RT-PCR)}

Total RNA was extracted using TRIzol RNA extraction kit (\#15596026) from Thermo Fisher Scientific, and then used to synthesize cDNA. Relative messenger RNA (mRNA) expression was analyzed with real time-quantitative PCR using the SYBR-Green mix from Thermo Fisher Scientific. The primer pairs for cDNA amplification were as follows: E-cadherin; (sense) 5'-AATAAAGACCAAGTGACCACC-3' and (antisense) 5'-GCAGAATCAGAATTAGCAAAGC-3', $\alpha$-catenin; (sense) 5'-ATGATCCCTGCTCTTCTGTG-3'and (antisense) 5'-GATACCATCT'TCCACAACTT'TCAG-3', vimentin; (sense) 5-GTGAATACCAAGACCTGGCTC-3' and (antisense) 5'-ATCCAGAT'TAGT'TTCCCTCAG-3', N-cadherin; (sense) 5'-TCATTAATGAGGGCCTTAAAGC-3' and (antisense) 5'-GT'TCAGGTAATCATAGTCCTGCT-3', and GAPDH as an internal control (sense) 5'-GAACCATGAGAAGTATGACAACAG-3' and (antisense) 5'-ATGGACTGTGGTCATGAGTC-3'.

\section{Immunoblotting analysis}

Lung adenocarcinoma cells were exposed to various treatments for $24 \mathrm{~h}$. Next, cells were harvested and lysed with cell lysis buffer (Bio-Rad), and protein concentration in the lysates was measured using the Bradford method (Alnuqaydan et al., 2020). Equal protein amount for each sample $(30 \mu \mathrm{g})$ was loaded into wells of pre-casted SDS-PAGE gel and resolved as per standard voltage, current, and time. Next, the proteins were transferred from the gel onto PVDF membrane for 
$1-2 \mathrm{~h}$ at 100 Volts. The membrane was blocked for nonspecific antigen binding sites with $5 \%$ fat-free milk in Tween-phosphate buffer saline (TBST). The blocked membrane was then incubated with a primary antibody (dissolved in the same blocking buffer) for $3 \mathrm{~h}$ at room temperature or overnight at $4^{\circ} \mathrm{C}$. Next, the membrane was washed with TBST and incubated with HRP-conjugated secondary antibody for $1 \mathrm{~h} 30 \mathrm{~min}$ at room temperature. The membrane was then washed with TBST dried, and incubated with enhanced chemiluminescence substance (Millipore) in dark for a couple of minutes at room temperature.. Using an X-ray film, the intensity of chemiluminescence was captured at different exposure times. The films were fixed and developed to analyze protein expression.

\section{Cell proliferation/cell viability assay}

The cells were cultured using the standard protocol as described previously (Dar et al., 2018). Briefly, lung adenocarcinoma cells were seeded in 96 -well plates at $5 \times 10^{3}$ per well and incubated at $37^{\circ} \mathrm{C}$ in a $5 \% \mathrm{CO}_{2}$ incubator overnight to allow for a proper adhesion to the bottom surface of the plate. Next morning fresh medium containing varying concentrations of paclitaxel was added to the cells for $24 \mathrm{~h}$ incubation. After completion of the time point, MTT dye was added to the wells at $2.5 \mathrm{mg} /$ $\mathrm{mL}$ for 3-4 h. The formazan crystals that had formed in the living cells were dissolved with DMSO and subjected to absorbance measurement at $570 \mathrm{~nm}$ wavelength. Cell viability was presented as a percentage of absorbance of the control cells not treated with paclitaxel.

\section{Transwell Boyden chamber invasion assay}

The assay is used to study the invasive potential of cancer cells (Rah et al., 2012). Briefly, $5 \times 10^{5}$ lung adenocarcinoma cells suspended in serum-free culture medium and placed in the upper insert of the Transwell Boyden chamber with a Matrigel bottom with pore size around $8 \mu \mathrm{m}$. The lower insert which contained 10\% FBS culture medium acted as a chemoattractant. After $24 \mathrm{~h}$ of incubation at $37^{\circ} \mathrm{C}$ in a $5 \% \mathrm{CO}_{2}$ incubator, the cells inside the upper part of the insert were removed with a cotton swab and the cells that migrated through Matrigel pore, attached to the bottom side of Matrigel insert, were fixed with ice-cold methanol for $15 \mathrm{~min}$, followed by $0.1 \%$ crystal violet staining for $10 \mathrm{~min}$. The Matrigel bottom insert was washed with PBS and analyzed under a light microscope to count the number of invasive cells per field.

\section{Wound healing assay}

The assay to study cell motility was performed as described previously (Sharma et al., 2012). Briefly, lung adenocarcinoma cells were seeded in a 6 -well plate at $5 \times 10^{5}$ cells per well and left overnight to adhere to the bottom of the wells. Next morning the cells were viewed with a microscope to check if $100 \%$ confluency was attained. After attaining $100 \%$ confluency, a wound was created by scratching over the monolayer of cells with a sterile $200 \mu \mathrm{L}$ microtip. After creating a wound, cells were washed with medium to remove detached cells and were photographed with the microscope with an inbuilt camera $(0 \mathrm{~h})$. After treating cells with varying concentrations of paclitaxel for $24 \mathrm{~h}$, cells were photographed again to analyze the migration of cells in the presence and absence of drugs $24 \mathrm{~h}$ after creating a wound.

\section{Apoptosis detection assay}

The cells seeded at $5 \times 10^{5}$ cells per well were exposed to paclitaxel treatment for $24 \mathrm{~h}$ or transfected with KDM5A plasmid or siRNA for $48 \mathrm{~h}$. Next the cells were stained for annexin V FITC and PI (Sigma Aldrich). Flow cytometry analysis was performed according to the manufacturer protocol in order to detect and quantify the percentage of the apoptotic cell population.

\section{Immunohistochemistry}

Immunohistochemistry staining was performed as described previously (Ahmad et al., 2017). The 4- $\mu \mathrm{m}$ thick sections were obtained using a microtome from the paraffin tissue blocks of lung adenocarcinoma and adjacent normal tissue obtained from lung adenocarcinoma patients. The tissue sections were properly adhered to glass slides and baked at $60^{\circ} \mathrm{C}$ for $1 \mathrm{~h}$ to melt paraffin. To detect KMD5A, Bond Polymer Refine Detection Kit for immunohistochemistry staining was used. The antigen retrieval was performed on the Bond III for 30 min with the help of Epitope retrieval solution 2. The slides were then incubated with KDM5A antibody (rabbit monoclonal) for $30 \mathrm{~min}$ and with an HRP-tagged secondary antibody for the next $10 \mathrm{~min}$. The visualization of $\mathrm{KD}$ M5A staining was performed by incubating the slides in 3,3'-diaminobenzidine for 5-10 min.. Finally, the counterstaining of slides was performed using hematoxylin and dehydration with low to high graded ethanol and xylene followed by application of airtight coverslip. The slides were stored at $4^{\circ} \mathrm{C}$ in dark until examined under the microscope.

\section{Statistical analysis}

All the data was analyzed using GraphPad Prism software. KDM5A expression in lung adenocarcinoma tissue samples and adjacent normal tissues was analyzed with the Chi-square test. Cell proliferation, apoptosis, and migration associated experiments results were analyzed with Student's $t$-test. All the experiments were performed at least three times. The value of $p \leq 0.05$ was considered statistically significant.

\section{RESULTS}

\section{Paclitaxel-resistance promotes cell proliferation in lung adenocarcinoma cells}

First, we assessed the impact of paclitaxel resistance on paclitaxel $\mathrm{IC}_{50}$ value in the cell lines. To this end, we treated a panel of lung adenocarcinoma cell lines (SK-LI-1, Calu-3, and A549 cells), including paclitaxelresistant Calu-3 cells (PTX-Calu-3), with varying concentrations of paclitaxel ( 0.1 to $10,000 \mathrm{nM})$ for $24 \mathrm{~h}$. We observed that lung adenocarcinoma cells (SK-LI-1, Calu3 , and A549 cells) treated with paclitaxel showed $50 \%$ cell death at an $\mathrm{IC}_{50}$ value of $(3.6 \pm 0.005,4.3 \pm 0.015$, and $4.5 \pm 0.106 \mathrm{nM}$ ), respectively (Fig. $1 \mathrm{~A}$ and $\mathrm{B}$ ). However, the $\mathrm{IC}_{50}$ value of paclitaxel-resistant PTX-Calu-3 cells was observed to be significantly higher $(7 \pm 0.176 \mu \mathrm{M})$. These results indicate that paclitaxel-resistance increases the $\mathrm{IC}_{50}$ value of paclitaxel. We hypothesize that paclitaxel resistance could also induce epigenetic modulation in the cells, possibly enhancing the aggressiveness of malignant cells, transforming differentiated cancer cells into undifferentiated cancer stem cells, which could enhance proliferation and metastases. 


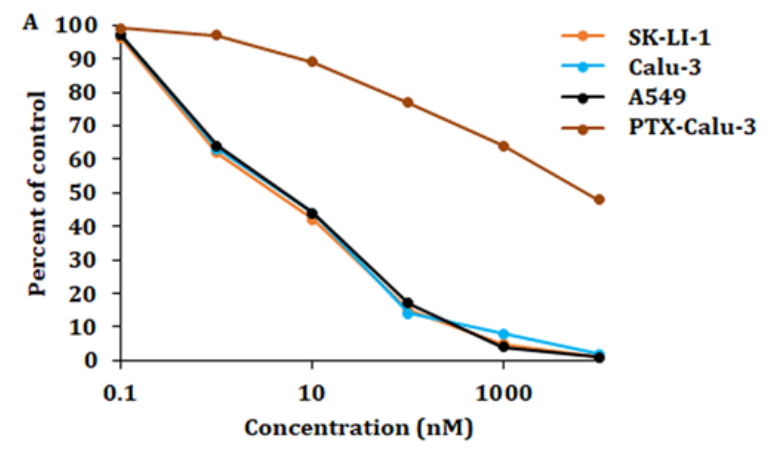

B

\begin{tabular}{cc}
\hline $\begin{array}{c}\text { Lung adenocarcinoma } \\
\text { Cell lines }\end{array}$ & $\mathrm{IC}_{50}$ Value \\
\hline SK-LI-1 & $3.6 \pm 0.005 \mathrm{nM}$ \\
\hline Calu-3 & $4.3 \pm 0.015 \mathrm{nM}$ \\
A549 & $4.5 \pm 0.106 \mathrm{nM}$ \\
PTX-Calu-3 & $7 \pm 0.176 \mu \mathrm{M}$ \\
\hline
\end{tabular}

Figure 1. Paclitaxel inhibits cell proliferation of lung adenocarcinoma cells.

(A) Antiproliferative effect of paclitaxel on the cell viability in lung adenocarcinoma cell lines (SK-LI-1, Calu-3, and A549) and in paclitaxel-resistant cancer cells (PTX-Calu-3) measured with the MTT assay. (B) Table of paclitaxel $I C_{50}$ values for lung adenocarcinoma cells (SK-LI-1, Calu-3, and A549) and paclitaxel-resistant cancer cells (PTX-Calu-3) treated with varying paclitaxel concentrations $(0.1$ to $10,000 \mathrm{nM})$ for $24 \mathrm{~h}$. The data represent the mean value \pm S.E. of three independent experiments $(n=3),{ }^{*} p<0.05$.
KDM5A-mediated paclitaxel resistance increases the expression P-Glycoprotein (P-gp) and cellular proliferation in lung adenocarcinoma cells.

Previous reports suggest that KDM5A expression significantly increases during the development of erlotinib and paclitaxel-resistance in lung adenocarcinoma and ovarian cancer (Stewart et al., 2015; Feng et al., 2017). Therefore, it is believed that higher expression of KDM5A plays a crucial role in the development of drug resistance in these types of cancer. We intended to evaluate whether paclitaxel-resistance could also increase the expression of KDM5A in lung adenocarcinoma cells. Our immunoblotting results showed a significantly higher expression of KDM5A in the cell lysate of paclitaxelresistant PTX-Calu-3 cells compared to paclitaxel-sensitive lung adenocarcinoma cell lines (SK-LI-1, Calu-3, and A549) (Fig. 2A). P-glycoprotein (P-gp) plays a critical role in drug transformation and its expression is elevated in drug resistance. To evaluate whether P-gp expression was elevated in paclitaxel-resistant PTX-Calu-3 cells, we used immunoblotting and we found higher expression of P-gp in paclitaxel-resistant PTX-Calu-3 cells compared to paclitaxel-sensitive lung adenocarcinoma cell lines (SK-LI-1, Calu-3, and A549). To further evaluate whether KDM5A expression had any association with P-gp expression in PTX-Calu-3 cells, we performed experiments where paclitaxel-sensitive lung adenocarcinoma cells (SKLI-1, Calu-3, and A549) were ectopically transfected with KDM5A plasmid. We also knocked down KDM5A in PTX-Calu-3 cells with siRNA for $48 \mathrm{~h}$. We found a significant increase in P-gp expression in paclitaxel-sensitive lung adenocarcinoma cells (SK-LI-1, Calu-3, and A549) ectopically transfected with KDM5A and a drastic decrease in the expression of P-gp in PTX-Calu-3 cells transfected with KDM5A siRNA (Figure 2B). To address whether the increased expression of KDM5A and

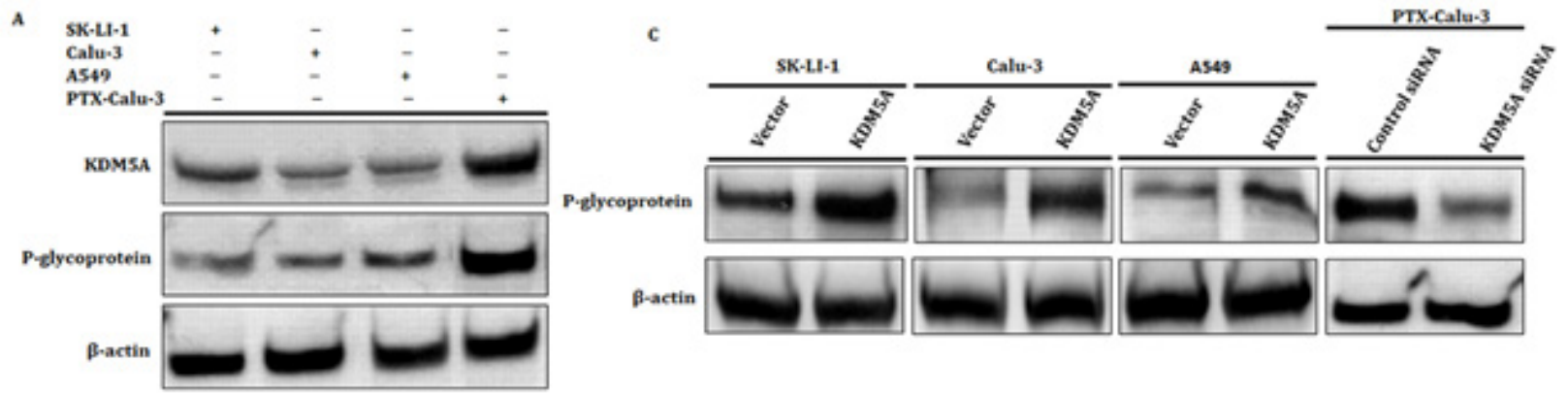

B

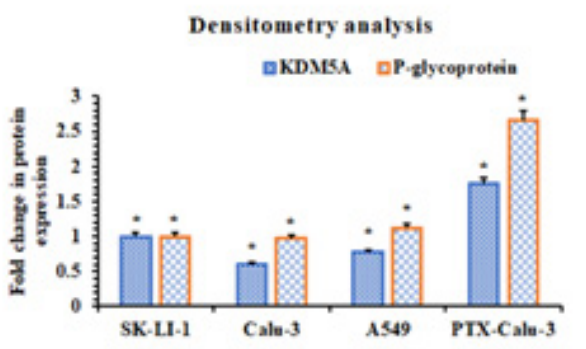

D

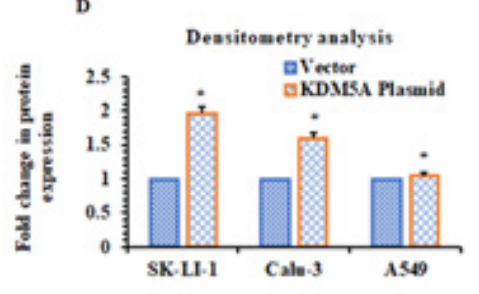

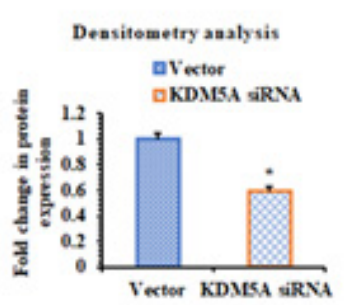

Figure 2. High expression of KDM5A in lung adenocarcinoma cells is correlated with p-glycoprotein protein levels and is associated with paclitaxel resistance.

(A) Immunoblotting showing KDM5A protein levels in lung adenocarcinoma (SK-LI-1, Calu-3, and A549) and paclitaxel-resistant lung adenocarcinoma (PTX-Calu-3) cell lines. (B) Fold change in protein expression calculated with densitometry analysis. (C) p-glycoprotein levels shown with immunoblotting in lung adenocarcinoma (SK-LI-1, Calu-3, and A549) and paclitaxel-resistant lung adenocarcinoma (PTXCalu-3) cells, transfected with KBM5A encoding plasmid or KDM5A siRNA. (D) Fold change in protein expression based on densitometric analysis. The data represent the mean value \pm S.E. of three independent experiments $(n=3),{ }^{*} p<0.05$. 

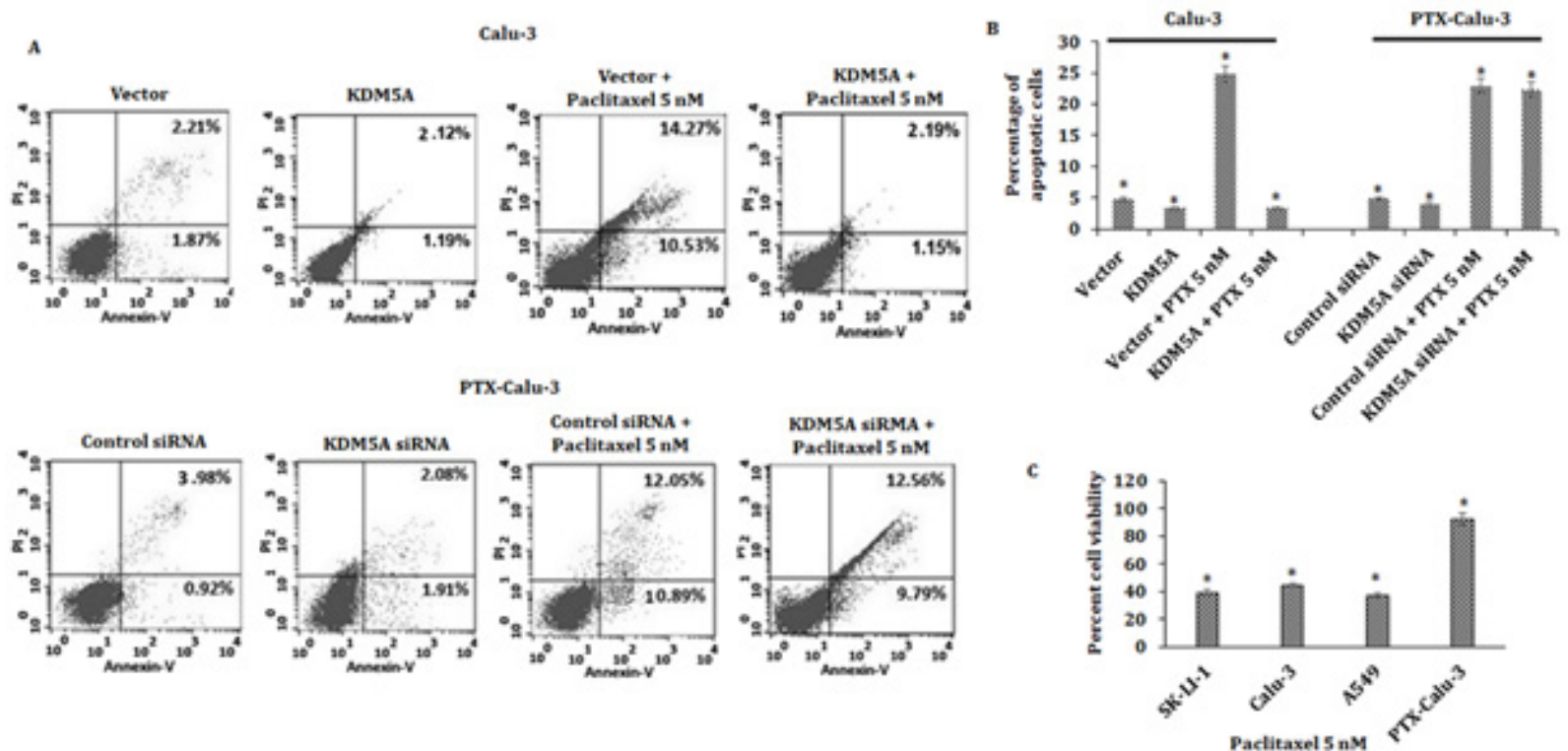

Figure 3. KDM5A modulates paclitaxel-resistance in lung adenocarcinoma cells.

(A) Flow cytometry based quantification of apoptotic cells in paclitaxel-sensitive lung adenocarcinoma (Calu-3) and paclitaxel-resistant lung adenocarcinoma (PTX-Calu-3) transfected for 48 hours with KDM5A plasmid or KDM5A siRNA and treated with $5 \mathrm{nM}$ paclitaxel for the last 24 h. (B) Bar diagram showing the quantification of the apoptotic population of cells with different settings (indicated). (C) Bar diagram showing the percentage of viable cells after $24 \mathrm{~h}, 5 \mathrm{nM}$ paclitaxel treatment. The data represent the mean value \pm S.E. of three independent experiments $(n=3),{ }^{*} p<0.05$.

P-gp could promote cell proliferation of paclitaxel-resistant PTX-Calu-3 when compared to lung adenocarcinoma cell lines (SK-LI-1, Calu-3, and A549 cells), we assessed cell viability. Our results demonstrated a significantly higher cell viability in paclitaxel-resistant PTX-Calu-3 than lung adenocarcinoma cell lines (SK-LI-1, Calu-3, and A549 cells) when exposed to paclitaxel treatment (5 nM) (Fig. 3C). Flow cytometry analysis of paclitaxel treated paclitaxel-sensitive lung adenocarcinoma cells (Calu-3) overexpressing KDM5A and paclitaxel-resistant PTX-Calu-3 cells with KDM5A knock-down, along with respective untreated controls, , revealed a significant decrease in apoptotic cell population in the cells transfected with KDM5A plasmid, and an increase in apoptotic cell population in the cells treated with KDM5A siRNA (Fig. 3A and B). Collectively, these results demonstrated that KDM5A-mediated paclitaxel-resistance increases the expression of P-gp and promotes cellular proliferation with the opposite effect on apoptosis.

\section{KDM5A-mediated paclitaxel resistance drives lung adenocarcinoma cells towards epithelial to mesenchymal transition (EMT) and increases tumor cell invasiveness and motility}

Numerous reports suggest that the development of drug resistance drives malignant cells towards EMT and thus helps to attain undifferentiated cancer stem cells. Since KDM5A mediates the development of paclitaxelresistance in lung adenocarcinoma, we intended to investigate whether paclitaxel-resistant cells have the ability to acquire transformation into mesenchymal cells via EMT. Our immunoblotting results showed that E-cadherin and $\alpha$-catenin expression was decreased in paclitaxel-resistant PTX-Calu-3 cells compared to lung adenocarcinoma cell lines (SK-LI-1, Calu-3, and A549 cells). Interestingly, we observed increased expression of mesenchymal markers $\mathrm{N}$-cadherin and vimentin in PTX-Calu-3 cells compared to SK-LI-1, Calu-3, and A549 cells (Fig. 4A). To validate whether KDM5A plays any role in EMT of paclitaxel-resistant lung adenocarcinoma cells, we designed two sets of experiments. In one set, paclitaxel-resistant PTX-Calu-3 cells were transfected with KDM5A siRNA for $48 \mathrm{~h}$. In the second set, lung adenocarcinoma cells (Calu-3) were ectopically transfected with KDM5A plasmid for $48 \mathrm{~h}$. Post-transfection, $48 \mathrm{~h}$, the expression of EMT markers in both sets was determined, both at the transcriptional level using RT-PCR and at the translational level by analyzing protein expression with immunoblotting. The knockdown of KDM5A in PTX-Calu-3 cells with siRNA significantly increased the expression of E-cadherin and $\alpha$-catenin, with a concomitant decrease in expression of mesenchymal markers $\mathrm{N}$-cadherin and vimentin, observed both at transcriptional and translational level (Fig. 4B). Conversely, KDM5A overexpression in lung adenocarcinoma cells (Calu-3) significantly increased mesenchymal markers $\mathrm{N}$-cadherin and vimentin expression, at the same time decreasing E-cadherin and $\alpha$-catenin expression both at transcriptional and translational level (Fig. 4C and D). Together, these results demonstrated KDM5A strongly modulates EMT in lung adenocarcinoma cells.

Various reports suggest that the transformation of malignant cells towards EMT is only one step away from metastasis. To investigate whether KDM5A plays a role in tumor cell invasion and motility, which are the crucial features in metastasis, we performed functional assays such as wound healing and Transwell Boyden chamber assay. Our wound healing results demonstrate that knockdown of KDM5A in paclitaxel-resistant PTX-Calu-3 cells significantly decreased migration of cells, as compared to control cells transfected with scramble siRNA (Fig. 5A and B). Similarly, Transwell Boyden chamber assay revealed that knockdown of KDM5A in PTX-Calu-3 cells significantly decreased the number of invasive cells that 


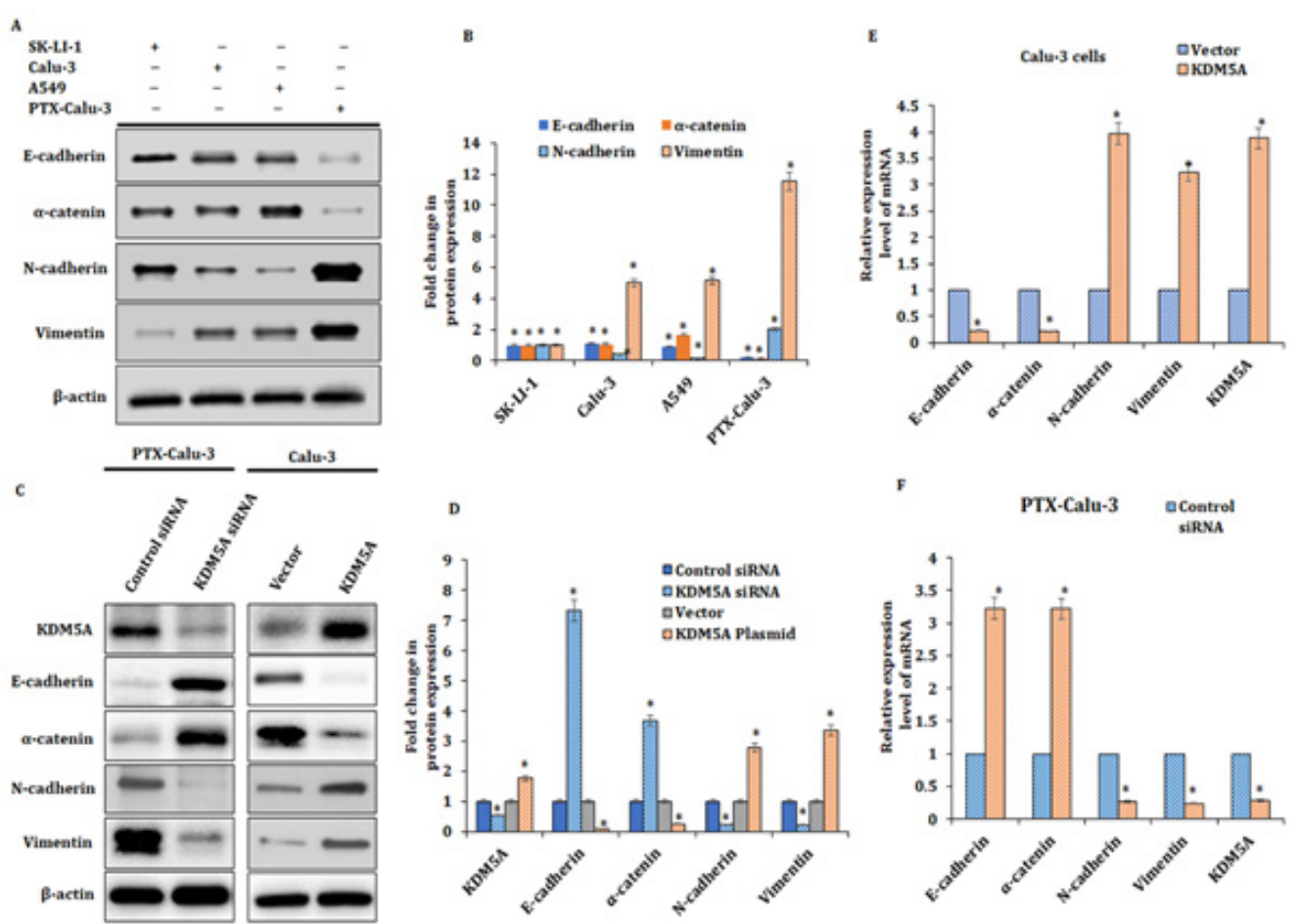

Figure 4. KDM5A drives lung adenocarcinoma cells towards EMT.

(A) Comparative analysis of EMT marker proteins expression (E-cadherin, a-cadherin, $\mathrm{N}$-cadherin, vimentin, along with loading control $\beta$-actin) by immunoblotting analysis of lung adenocarcinoma (SK-LI-1, Calu-3, and A549) and paclitaxel-resistant lung adenocarcinoma (PTX-Calu-3) cell extracts. (B) Fold change in protein expression in densitometry analysis. (C) Protein expression of KDM5A and EMT markers (E-cadherin, a-cadherin, $\mathrm{N}$-cadherin, vimentin along loading control $\beta$-actin) in paclitaxel-sensitive (Calu-3) and paclitaxel-resistant (PTX-Calu-3) cells 48 hours after transfection with KDM5A plasmid or KDM5A siRNA, shown with immunoblotting. (D) Fold change in protein expression based on densitometric analysis. (E and F) mRNA expression of EMT markers in paclitaxel-sensitive (Calu-3) and paclitaxel-resistant (PTX-Calu-3) lung adenocarcinoma cells after transient transfection with KDM5A encoding plasmid or KDM5A siRNA for $48 \mathrm{~h}$. The data represent the mean value \pm S.E. of three independent experiments $(n=3),{ }^{*} p<0.05$.

$A$

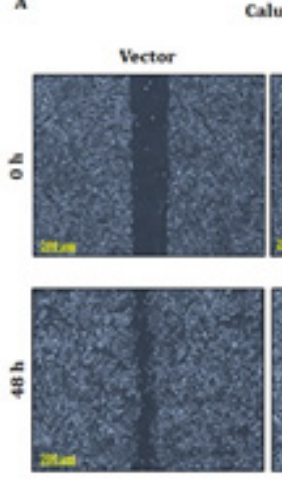

c
Calu-3 3
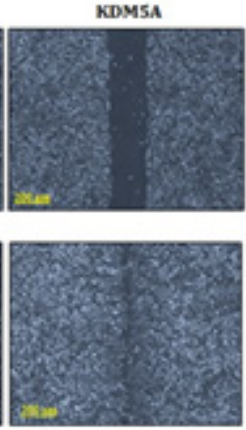

Calu-3

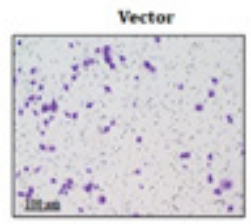

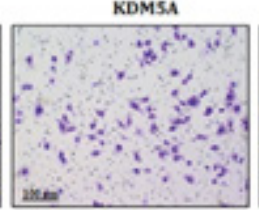

PTX-Calu-3

Control siRNa
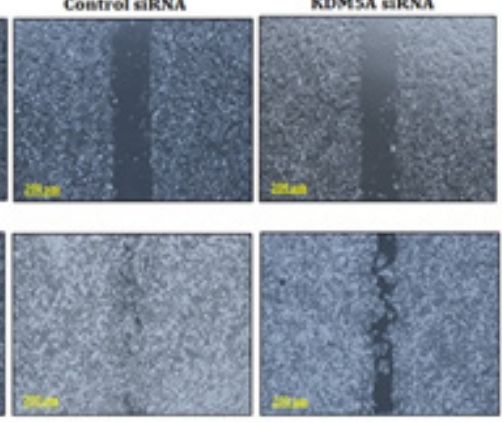

PTX-Cale-3

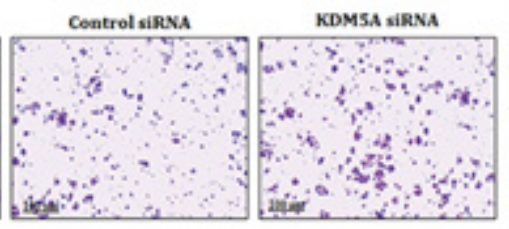

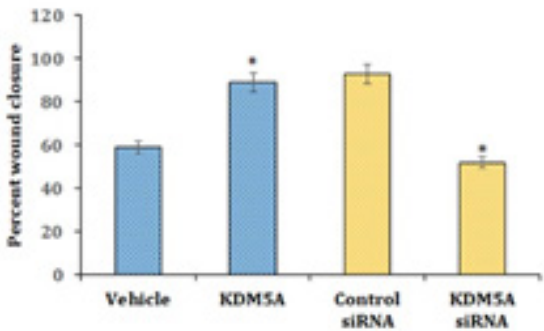

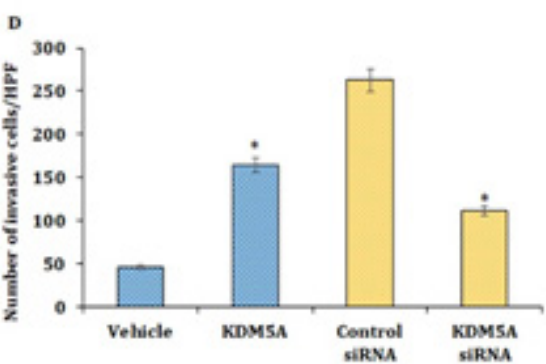

Figure 5. KDM5A increases the motility and invasion of lung adenocarcinoma cells.

(A) Wound healing assay was performed to determine the motility of paclitaxel-sensitive and paclitaxel-resistant lung adenocarcinoma cells that were transiently transfected with KDM5A encoding plasmid or KDM5A siRNA, respectively, for 48 h. Scale bar: 200 4 m; $10 x$. (B) Bar diagram showing the percentage of wound closure 48 hours after transfection . (C) Transwell Boyden chamber assay was performed to determine the invasive potential of paclitaxel-sensitive and paclitaxel-resistant lung adenocarcinoma cells that were transiently transfected with KDM5A encoding plasmid or KDM5A siRNA, respectively, for 48 h. Scale bar: $100 \mu \mathrm{m}$; 20x. (D) Bar diagram showing the number of invasive cells paclitaxel-sensitive and paclitaxel-resistant lung adenocarcinoma cells 48 hours post-transfection. The data represent the mean value \pm S.E. of three independent experiments $(n=3),{ }^{*} p<0.05$. 
passed through porous Matrigel membrane, as compared to control. Conversely, KDM5A overexpression significantly increased the number of invasive tumor cells passing through porous Matrigel membrane (Fig. 5C and D). Collectively, these results demonstrate that KDM5A not only plays a critical role in driving malignant cells towards EMT but can also facilitate tumor cell metastasis in paclitaxel-resistant lung adenocarcinoma cells.

\section{High expression of KDM5A has a strong causal association with paclitaxel-resistance in lung adenocarcinoma}

The major driver of tumor progression is deregulation at the gene level. Recent reports suggest that the gain of function of various protooncogenes promotes tumorigenesis. Previous reports suggest that the amplification of KDM5A has a strong causal association with tumor development in many malignancies such as breast and ovarian cancer. To examine whether KDM5A plays a role in lung adenocarcinoma, we have collected 87 confirmed lung adenocarcinoma tissue samples along with adjacent normal tissue samples from the patients. After extracting RNA from all tissue samples, RT-PCR was performed to determine the expression levels of KDM5A. Our results showed a significantly higher expression of KDM5A in lung adenocarcinoma tissues compared to adjacent normal tissues (Fig. 6B). Furthermore, immunohistochemistry analysis revealed high expression of KDM5A in lung adenocarcinoma tissues compared to adjacent normal tissues (Fig. 6A). Additionally, we also observed significantly higher expression of KDM5A both at transcriptional as well as translational level in paclitaxel-resistant lung adenocarcinoma cells (PTX-Calu-3) compared to paclitaxel-sensitive lung adenocarcinoma cell lines (SK-LI-1, Calu-3, and A549 cells) (Fig. 2A).

\section{DISCUSSION}

For the last two decades, the burden of lung cancer has been rising. Currently, lung cancer accounts for the highest number of cancer mortality in both genders and poses a serious threat to human health (Bray et al., 2018). Despite various novel chemotherapeutic drug combinations that were tested along with the surgical resection, the 5-year survival rate remains low in lung cancer (Mamdani et al., 2015). The primary reason for the low 5-year survival rate is tumor heterogeneity and the development of drug resistance against the present regiment of chemotherapeutic drugs (Xue et al., 2017). Thus, there is an urgent need to investigate the underlying tumor heterogeneity, resistance to existing chemotherapeutic drugs, and development of novel antitumor agents, and new chemotherapeutic combinations to curtail lung cancer.

Previous reports suggest that genetic mutations to protooncogenes play a critical role in the development of tumor heterogeneity and drug resistance (Pathania et al., 2016). Recent reports suggest that epigenetic modulations such as histone modifications modulate the expression of some key proteins and helps in the development of drug resistance (Rastgoo et al., 2017). One of the key histone demethylases, KDM5A, regulates numerous genes expression by catalyzing histone $\mathrm{H} 3 \mathrm{~K} 4$ demethylation and thereby regulates cellular processes (Johansson et al., 2016). Previous reports suggest that KDM5A acts as oncogenic driver by binding to tumor suppressor retinoblastoma-binding protein and in turn negating its tumor-suppressive role in retinoblastoma (Blair et al., 2011; Hookway 2016). Recent studies reveal that in some malignancies such as gastric cancer and acute myeloid cancer, the expression of KDM5A is significantly elevated (Shokri et al., 2018). Aberrant expression of KDM5A in various cancers such as breast cancer, ovarian cancer, and glioblastoma have been reported to play a critical role in the development of drug resistance (Xhabija \& Kidder 2019).

Recent reports suggest the involvement of KDM5A in breast cancer metastasis to the lung and in the development of drug resistance in ovarian cancer (Hou et al., 2012; Feng et al., 2017). However, the role of KD-

A
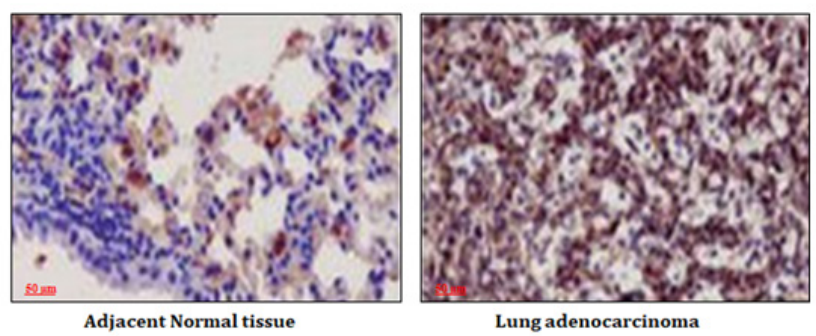

B

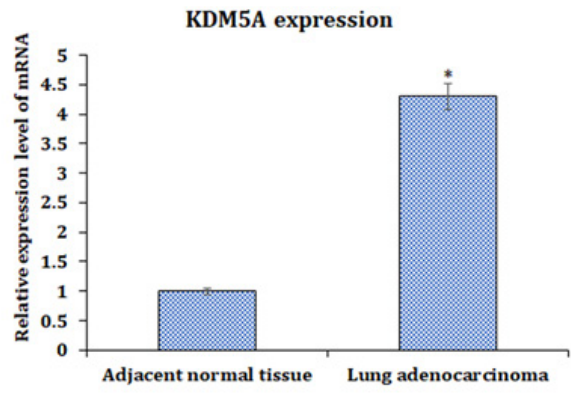

Figure 6. High expression of KDM5A in lung adenocarcinoma tissues.

(A) Immunohistochemical staining showing high expression of KDM5A in lung adenocarcinoma compared to normal adjacent tissue. Scale bar: $50 \mu \mathrm{m}$; 20x. (B) RT-PCR analysis showed significantly higher expression of KDM5A mRNA in lung adenocarcinoma compared to adjacent normal tissue. The data represent the mean value \pm S.E. of three independent experiments $(n=3),{ }^{*} p<0.05$. 
M5A in lung adenocarcinoma is yet to be evaluated. In the current study, we observed that KDM5A was highly expressed in paclitaxel-resistant lung adenocarcinoma cell line (PTX-Calu-3) and acted as a growth kinetics driver upon paclitaxel treatment, as shown via elevated $\mathrm{IC}_{50}$ values compared to paclitaxel-sensitive lung adenocarcinoma cells (SK-LI-1, Calu-3, and A549 cells). Further, we found that high expression of KDMA5 stimulates EMT, motility, and invasion in lung adenocarcinoma cells. Additionally, we found high expression of KDM5A in tissue samples of patients who were on paclitaxel chemotherapy, which suggests a casual association of KDM5A with acquiring paclitaxel resistance in lung adenocarcinoma. Taken together, these results suggest that KDM5A could be a novel drug target in paclitaxel drug resistance in lung adenocarcinoma.

Despite significant efficiency of the existing chemotherapeutic drug regime in lung cancer patients, a group of patients $(25-30 \%)$ have an intrinsic ability to develop drug resistance due to protooncogenic mutations within the EGFR gene (Yano et al., 2011; Coyle et al., 2017). The development of drug resistance negates any chemotherapeutic effect of the drug and leads to activation of cellular proliferation, drives proliferating cells towards EMT and spread of metastases to distinct sites or organs (Barker et al., 2015; Timaner et al., 2020). In this study, we first addressed the effect of paclitaxel treatment on both paclitaxel-sensitive and paclitaxel-resistant lung adenocarcinoma cell lines (SK-LI-1, Calu-3, A549 cells and PTX-Calu-3). Consistent with previous studies, we observed significantly increased paclitaxel $\mathrm{IC}_{50}$ value $(7 \pm 0.176 \mu \mathrm{M})$ in paclitaxel-resistant PTX-Calu-3 cells compared to SK-LI-1, Calu-3, and A549 cells (3.6 \pm 0.005 , $4.3 \pm 0.015$, and $4.5 \pm 0.106 \mathrm{nM})$. These results indicate that the development of chemotherapeutic drug resistance not only sustains the tumor cells, but may also accelerate cell proliferation, possibly by activating cell cycle regulatory proteins and nullifying cell cycle checkpoints.

Mounting evidence suggests that aberrant expression of histone demethylases has a strong causal association with tumorigenesis (Jin \& Robertson, 2013). Previous reports suggest that KDM5A expression significantly increases during the development of erlotinib and paclitaxel-resistance in lung adenocarcinoma and ovarian cancer, respectively (Hou et al., 2012; Jin \& Robertson, 2013; Feng et al., 2017). Consistent with the abovementioned studies, our immunoblotting results showed a significantly higher expression of KDM5A in paclitaxelresistant lung adenocarcinoma cells (PTX-Calu-3) when compared to paclitaxel-sensitive lung adenocarcinoma cell lines (SK-LI-1, Calu-3, and A549 cells). The development of multidrug resistance (MDR) is one of the primary impediments to the effective chemotherapeutics of anticancer drugs (Nikolaou et al., 2018). Reports suggest that drug transporter P-glycoprotein (P-gp) is a vital player in the MDR to chemotherapeutic drugs (Shukla et al., 2011; Sun et al., 2012). A member of the ATPbinding cassette (ABC)-transport family, P-gp, whose expression is elevated in tumor cells, utilizes energy in the form of ATP to export drug molecules out of the tumor cell (Sun et al., 2012). Linn et al., demonstrated that the P-gp expression significantly increases in colorectal tumors and in adjacent mucosal cells and confers drug resistance to anti-tumor drugs (Linn \& Giaccone, 1995). Consistent with the above results, we found a significantly elevated expression of P-gp in paclitaxel-resistant lung adenocarcinoma cells (PTX-Calu-3) cells compared to drug-sensitive lung adenocarcinoma cells (SK-LI-1, Calu-3, and A549 cells). In the further validation, our overexpression and knockdown experiments clearly demonstrated that KDM5A expression influences the expression of P-gp, which in turn confers paclitaxel resistance in lung adenocarcinoma cells. Furthermore, an increase in the expression of KDM5A and P-gp decreases apoptosis by expelling paclitaxel molecules out of the cells, thereby enhancing the proliferation of paclitaxel-resistant lung adenocarcinoma cells.

KDM5A plays a crucial role in the development of drug-resistance and impedes the development of successful chemotherapy, both traditional and targeted (Lackner et al., 2012). Recent reports suggest that the drugresistant malignant cells have a strong intrinsic ability to induce tumor heterogeneity and allow cells to undergo EMT, which in turn leads to development of undifferentiated cancer stem cells (Lee et al., 2016; Testa et al., 2018). The development of this type of cells leads to relatively high resistance to chemotherapeutic drugs and disturbances in tumor cell apoptosis (Baguley, 2010). Feng and others (Feng et al., 2017) demonstrated that high KDM5A expression in ovarian cancer promotes paclitaxel-mediated drug resistance and switches the polarized epithelial cells towards the mesenchymal phenotypic morphology. Consistent with the previous findings, our results also demonstrate that KDM5A-mediated paclitaxel-resistant lung adenocarcinoma cells acquire the EMT phenotype by increasing mesenchymal markers $\mathrm{N}$ cadherin and vimentin, and decreasing E-cadherin and $\alpha$-cadherin, both at transcriptional and translational level. Previous reports suggest that epigenetic modulations play a key role in driving tumor cells towards EMT leading to their spreading to distinct sites as metastatic cells (Tsai \& Yang 2013; Chatterjee et al., 2018). Interestingly, aberrant expression of KDM5A has been reported to have a key role in driving tumor cells towards EMT and activating their motility and migration in various malignancies such as ovarian, breast, and gastric cancer (Zhou \& Kopeček 2013; Tachtsidis et al., 2016; Qiu et al., 2018). In agreement with the above-mentioned findings, our results also demonstrated that overexpression and knockdown of KDM5A significantly influenced the motility and invasiveness of lung adenocarcinoma cells. Together, these findings suggest that besides having a crucial role in driving tumor cells towards EMT, KDM5A may further push the cells to de-undifferentiated into cancer stem cell. The dysregulated of gene expression has been shown to be involved in the progression of tumorigenesis (Sawan et al., 2008). Recent reports suggest that the gain of function of various protooncogenes promotes tumorigenesis to counter the chemotherapeutic effects and stimulates development of the drug resistant tumor cells (Igney \& Krammer, 2002). Studies have shown the involvement of KDM5A in development of several human malignancies which include but are not limited to breast, gastric and ovarian cancer (Rasmussen \& Staller, 2014; Plch et al., 2019). Consistent with previous results, our findings showed a significantly higher expression of KDM5A in lung adenocarcinoma tissues and paclitaxelresistant lung adenocarcinoma cells (PTX-Calu-3) compared to adjacent normal tissues and to paclitaxel-sensitive lung adenocarcinoma cell lines (SK-LI-1, Calu-3, and A549 cells), respectively. Collectively, these findings showed that aberrant expression of KDM5A underlay paclitaxel-mediated drug resistance, promoted EMT, and suggest that it can increase cell viability, enhance activation of protooncogenes and promote dissemination metastatic cells to distant sites.

In conclusion, the current study demonstrated elevated expression of KDM5A in lung adenocarcinoma 
tissues when compared to adjacent normal tissues. Our results demonstrate that KDM5A acts as a growth driver coinciding with elevated $\mathrm{IC}_{50}$ values in paclitaxel-resistant PTX-Calu-3 cells compared to paclitaxel-sensitive lung adenocarcinoma cells (SK-LI-1, Calu-3, and A549). Furthermore, we found that high expression of KDMA5 stimulates EMT, motility, invasion, and may thus promote metastasis in lung adenocarcinoma. Taken together, these results suggest that high expression of KDM5A has a causal association with paclitaxel resistance in lung adenocarcinoma and could be a novel drug target in paclitaxel-mediated drug resistance in lung adenocarcinoma.

\section{Authors Contributions}

Lidong Xu: conceptualization, visualization, data curation, acquisition, formal analysis. Hong Wu: conceptualization, writing, review, and editing. Xun Hu: conceptualization, design of experiments, writing, editing, and making the original draft of the manuscript.

Conflict of interest

The authors declare no conflict of interest.

\section{REFERENCES:}

Ahmad R, Rah B, Bastola D, Dhawan P, Singh AB (2017) Obesityinduces organ and tissue specific tight junction restructuring and barrier deregulation by claudin switching. Sci. Rep. 7: 1-16. https:// doi.org/10.1038/s41598-017-04989-8

Alnuqaydan AM, Rah B, Almutary AG, Chauhan SS (2020) Synergistic antitumor effect of 5-fluorouracil and withaferin-A induces endoplasmic reticulum stress-mediated autophagy and apoptosis in colorectal cancer cells. Am. J. Cancer Res. 3: 799-815

Alsaab HO, Sau S, Alzhrani R, Tatiparti K, Bhise K, Kashaw SK, Iyer AK (2017) PD-1 and PD-L1 checkpoint signaling inhibition for cancer immunotherapy: mechanism, combinations, and clinical outcome. Front. Pharmacol. 8: 561. https://doi.org/10.3389/ fphar.2017.00561

Baguley BC (2010) Multiple drug resistance mechanisms in cancer. Mol. Biotechnol. 46: 308-316. https://doi.org/10.1007/s12033-010-9321-2

Barker HE, Paget JT, Khan AA, Harrington KJ (2015) The tumour microenvironment after radiotherapy: mechanisms of resistance and recurrence. Nat. Rev. Cancer 15: 409-425. https://doi.org/10.1038/ nrc3958

Blair LP, Cao J, Zou MR, Sayegh J, Yan Q (2011) Epigenetic regulation by lysine demethylase 5 (KDM5) enzymes in cancer. Cancers 3: 1383-1404. https://doi.org/10.3390/cancers3011383

Brabletz T, Kalluri R, Nieto MA, Weinberg RA (2018) EMT in cancer. Nat. Rev. Cancer 18: 128. https://doi.org/10.1038/nrc.2017.118

Bray F, Ferlay J, Soerjomataram I, Siegel RL, Torre LA, Jemal A (2018) Global cancer statistics 2018: GLOBOCAN estimates of incidence and mortality worldwide for 36 cancers in 185 countries. CA: Cancer J. Clin. 68: 394 424. https://doi.org/10.3322/caac.21492

Cao J, Liu Z, Cheung WK, Zhao M, Chen SY, Chan SW, Booth CJ, Nguyen DX, Yan Q (2014) Histone demethylase RBP2 is critical for breast cancer progression and metastasis. Cell Rep. 6: 868-877. https://doi.org/10.1016/j.celrep.2014.02.004

Cedar H, Bergman Y (2009) Linking DNA methylation and histone modification: patterns and paradigms. Nat. Rev. Genet. 10: 295-304. https://doi.org/10.1038/nrg2540.

Chatterjee A, Rodger EJ, Eccles MR (2018) Epigenetic drivers of tumourigenesis and cancer metastasis. In Seminars in cancer biology, pp 149-159. Elsevier. https://doi.org/10.1016/j.semcancer.2017.08.004

Coyle KM, Boudreau JE, Marcato P (2017) Genetic mutations and epigenetic modifications: driving cancer and informing precision medicine. BioMed Res. Int. 2017: 9620870. https://doi. org/10.1155/2017/9620870

Dar AM, Rah B, Mir S, Nabi R, Gatoo MA, Mashrai A, Khan Y (2018). DNA binding, artificial nuclease activity and cytotoxic studies of newly synthesized steroidal pyrimidines. Int. J. Biol. Macromol. 111: 52-61. https://doi.org/10.1016/j.ijbiomac.2017.12.128

de Groot PM, Wu CC, Carter BW, Munden RF (2018) The epidemiology of lung cancer. Transl. Lung Cancer Res. 7: 220-233. https:// dx.doi.org/10.21037\%2Ftlcr.2018.05.06

Dinglin XX, Huang Y, Liu H, Zeng YD, Hou X, Chen LK (2013) Pemetrexed and cisplatin combination with concurrent whole brain radiotherapy in patients with brain metastases of lung adenocarcinoma: a single-arm phase II clinical trial. J. Neuro-Oncol. 112: 461-466. https://doi.org/10.1007/s11060-013-1079-5

Feng T, Wang Y, Lang Y, Zhang Y (2017) KDM5A promotes proliferation and EMT in ovarian cancer and closely correlates with PTX resistance. Mol. Med. Rep. 16: 3573-3580. https://doi.org/10.3892/ $\mathrm{mmr} .2017 .6960$

Gandhi L, Rodríguez-Abreu D, Gadgeel S, Esteban E, Felip E, De Angelis F, Domine M, Clingan P, Hochmair MJ, Powell SF (2018) Pembrolizumab plus chemotherapy in metastatic non-small-cell lung cancer. New Eng. J. Med. 378: 2078-2092. https://doi.org/10.1056/ NEJMoa1801005

Hookway E (2016) The role of the lysine demethylases KDM5 and KDM6 in bone malignancies. University of Oxford

Hou J, Wu J, Dombkowski A, Zhang K, Holowatyj A, Boerner JL, Yang ZQ (2012) Genomic amplification and a role in drug-resistance for the KDM5A histone demethylase in breast cancer. Am. J. Transl. Res. 4: 247

Hu D, Jablonowski C, Cheng PH, AlTahan A, Li C, Wang Y, Palmer L, Lan C, Sun B, Abu-Zaid A, Fan Y (2018) KDM5A regulates a translational program that controls p53 protein expression. Iscience $\mathbf{9}$ 84-100. https://doi.org/10.1016/j.isci.2018.10.012

Huang CY, Ju DT, Chang CF, Reddy PM, Velmurugan BK (2017) A review on the effects of current chemotherapy drugs and natural agents in treating non-small cell lung cancer. Biomedicine 7: 23. https://dx.doi.org/10.1051\%2Fbmdcn\%2F2017070423

Igney FH, Krammer PH (2002) Death and anti-death: tumour resistance to apoptosis. Nat. Rev. Cancer 2: 277-288. https://doi. org/10.1038/nrc776

Jin B, Robertson KD (2013) DNA methyltransferases, DNA damage repair, and cancer. In Epigenetic Alterations in Oncogenesis pp 3-29. Springer. https://doi.org/10.1007/978-1-4419-9967-2_1

Johansson C, Velupillai S, Tumber A, Szykowska A, Hookway ES, Nowak RP, Strain-Damerell C, Gileadi C, Philpott M, BurgessBrown N, Wu N (2016) Structural analysis of human KDM5B guides histone demethylase inhibitor development. Nat. Chem. Biol. 12: 539-545. https://doi.org/10.1038/nchembio.2087

Kim ST, Cristescu R, Bass AJ, Kim KM, Odegaard JI, Kim K, Liu XQ, Sher X, Jung H, Lee M, Lee S (2018) Comprehensive molecular characterization of clinical responses to PD-1 inhibition in metastatic gastric cancer. Nat. Med. 24: 1449-1458. https://doi. org/10.1038/s41591-018-0101-z

Kolybaba A, Classen AK (2014) Sensing cellular states - signaling to chromatin pathways targeting Polycomb and Trithorax group function. Cell Tissue Res. 356: 477-493. https://doi.org/10.1007/s00441014-1824-x

Kooistra SM, Helin K (2012) Molecular mechanisms and potential functions of histone demethylases. Nat. Rev. Mol. Cell Biol. 13: $297-$ 311. https://doi.org/10.1038/nrm3327

Lackner MR, Wilson TR, Settleman J (2012) Mechanisms of acquired resistance to targeted cancer therapies. Future Oncol. 8: 999-1014. https://doi.org/10.2217/fon.12.86

Lee G, Hall III RR, Ahmed AU (2016) Cancer stem cells: cellular plasticity, niche, and its clinical relevance. J. Stem Cell Res Ther. 6: 363. https://dx.doi.org/10.4172\%2F2157-7633.1000363

Li Z, Qing Y, Guan W, Li M, Peng Y, Zhang S, Xiong Y, Wang D (2014) Predictive value of APE1, BRCA1, ERCC1 and TUBB3 expression in patients with advanced non-small cell lung cancer (NSCLC) receiving first-line platinum-paclitaxel chemotherapy. Cancer Chemother. Pharmacol. 74: 777-786. https://doi.org/10.1007/ s00280-014-2562-1

Linn S, Giaccone G (1995) MDR1/P-glycoprotein expression in colorectal cancer. Eur. J. Cancer 31: 1291-1294. https://doi. org/10.1016/0959-8049(95)00278-Q

Mamdani H, Induru R, Jalal SI (2015) Novel therapies in small cell lung cancer. Transl. Lung Cancer Res. 4: 533-544. https://doi. org/10.3978\%2Fj.issn.2218-6751.2015.07.20

Minguet J, Smith KH, Bramlage P (2016) Targeted therapies for treatment of non-small cell lung cancer - Recent advances and future perspectives. Int. J. Cancer 11: 2549-2561. https://doi.org/10.1002/ ijc. 29915

Nagano T, Tachihara M, Nishimura Y (2018) Mechanism of resistance to epidermal growth factor receptor-tyrosine kinase inhibitors and a potential treatment strategy. Cells 11: 212. https://doi.org/10.3390/ cells 7110212

Nikolaou M, Pavlopoulou A, Georgakilas AG, Kyrodimos E (2018) The challenge of drug resistance in cancer treatment: a current overview. Clin. Exp. Metastasis. 35: 309-318. https://doi.org/10.1007/ s10585-018-9903-0

Oser MG, Sabet AH, Gao W, Chakraborty AA, Schinzel AC, Jennings RB, Fonseca R, Bonal DM, Booker MA, Flaifel A, Novak JS (2019) The KDM5A/RBP2 histone demethylase represses NOTCH signaling to sustain neuroendocrine differentiation and promote small cell lung cancer tumorigenesis. Genes Dev. 33: 1718-1738. http://www. genesdev.org/cgi/doi/10.1101/gad.328336.119

Pathania R, Ramachandran S, Mariappan G, Thakur P, Shi H, Choi JH, Manicassamy S, Kolhe R, Prasad PD, Sharma S, Lokeshwar BL (2016) Combined inhibition of DNMT and HDAC blocks the tumorigenicity of cancer stem-like cells and attenuates mammary tumor growth. Cancer Res. 76: 3224-3235. https://doi.org/ 10.1158/00085472 . 
Plch J, Hrabeta J Eckschlager T (2019) KDM5 demethylases and their role in cancer cell chemoresistance. Int. J. Cancer 144: 221-231. https://doi.org/10.1002/ijc.31881

Qiu Z, Wang J Wu Y (2018) The landscape of histone modification in cancer metastasis. In Cancer Metastasis pp 39. IntechOpen

Rah B, Amin H, Yousuf K, Khan S, Jamwal G, Mukherjee D, Goswami A (2012) A novel MMP-2 inhibitor 3-azidowithaferin A (3-azidoWA) abrogates cancer cell invasion and angiogenesis by modulating extracellular Par-4. PloS One 7: e44039. https://doi.org/10.1371/ journal.pone.0044039

Rasmussen PB, Staller P (2014) The KDM5 family of histone demethylases as targets in oncology drug discovery. Epigenomics 6: 277-286. https://doi.org/10.2217/epi.14.14

Rastgoo N, Abdi J, Hou J, Chang H (2017) Role of epigenetics-microRNA axis in drug resistance of multiple myeloma. J. Hematol. Oncol. 10: 121. https://doi.org/10.1186/s13045-017-0492-1

Roointan A, Mir TA, Wani SI, Hussain KK, Ahmed B, Abrahim S, Savardashtaki A, Gandomani G, Gandomani M, Chinnappan R, Akhtar MH (2019) Early detection of lung cancer biomarkers through biosensor technology: A review. J. Pharm. Biomed. Anal. 164: 93-103. https://doi.org/10.1016/j.jpba.2018.10.017

Sawan C, Vaissière T, Murr R, Herceg Z (2008) Epigenetic drivers and genetic passengers on the road to cancer. Mutat. Res. 642: 1-13. https://doi.org/10.1016/j.mrfmmm.2008.03.002

Serrano-Gomez SJ, Maziveyi M, Alahari SK (2016) Regulation of epithelial-mesenchymal transition through epigenetic and post-translational modifications. Mol. Cancer 15: 1-14. https://doi.org/10.1186/ s12943-016-0502-x.

Sharma DK, Rah B, Lambu MR, Hussain A, Yousuf SK, Tripathi AK, Singh B, Jamwal G, Ahmed Z, Chanauria N, Nargotra A (2012) Design and synthesis of novel N, N'-glycoside derivatives of 3 , 3'-diindolylmethanes as potential antiproliferative agents. MedChemComm. 3: 1082-1091

Shen YA, Li WH, Chen PH, He CL, Chang YH, Chuang CM (2017) Intraperitoneal delivery of a novel liposome-encapsulated paclitaxel redirects metabolic reprogramming and effectively inhibits cancer stem cells in Taxol ${ }^{\circledR}$-resistant ovarian cancer. Am. J. Transl. Res. 7: $841-855$

Shokri G, Doudi S, Fathi-Roudsari M, Kouhkan F, Sanati MH (2018) Targeting histone demethylases KDM5A and KDM5B in AML cancer cells: A comparative view. Leukemia Res. 68: 105-111. https:// doi.org/10.1016/j.leukres.2018.02.003
Shukla S, Ohnuma S, V Ambudkar S (2011) Improving cancer chemotherapy with modulators of ABC drug transporters. Curr. Drug Targets 12: 621-630. https://doi.org/10.2174/138945011795378540

Stewart EL, Tan SZ, Liu G, Tsao MS (2015) Known and putative mechanisms of resistance to EGFR targeted therapies in NSCLC patients with EGFR mutations - a review. Transl. Lung Cancer Res. 4: 67-81. https://dx.doi.org/10.3978\%2Fj.issn.2218-6751.2014.11.06

Sun YL, Patel A, Kumar P, Chen ZS (2012) Role of ABC transporters in cancer chemotherapy. Chin. J. Cancer 31: 51-57. https://dx.doi. org $/ 10.5732 \% 2$ Fcjc.011.10466

Tachtsidis A, McInnes LM, Jacobsen N, Thompson E, Saunders CM (2016) Minimal residual disease in breast cancer: an overview of circulating and disseminated tumour cells. Clin. Exp. Metastasis 33: 521-550. https://doi.org/10.1007/s10585-016-9796-8

Testa U, Petrucci E, Pasquini L, Castelli G, Pelosi E (2018) Ovarian cancers: genetic abnormalities, tumor heterogeneity and progression, clonal evolution and cancer stem cells. Medicines 5: 16. https://doi. org /10.3390/medicines5010016

Timaner M, Tsai KK, Shaked Y (2020) The multifaceted role of mesenchymal stem cells in cancer. In Seminars in Cancer Biology, pp 225237. Elsevier. https://doi.org/10.1016/j.semcancer.2019.06.003

Tsai JH, Yang J (2013) Epithelial-mesenchymal plasticity in carcinoma metastasis. Genes Dev. 27: 2192-2206. http://www.genesdev.org/ cgi/doi/10.1101/gad.225334.113

Xhabija B, Kidder BL (2019) KDM5B is a master regulator of the H3K4-methylome in stem cells, development and cancer. In Seminars in Cancer Biology, pp 79-85. Elsevier. https://doi.org/10.1016/j. semcancer.2018.11.001

Xue Y, Hou S, Ji H, Han X (2017) Evolution from genetics to phenotype: reinterpretation of NSCLC plasticity, heterogeneity, and drug resistance. Protein Cell. 8: 178-190. https://doi.org/10.1007/s13238016-0330-1

Yano S, Wang W, Li Q, Yamada T, Takeuchi S, Matsumoto K, Nishioka Y, Sone S (2011) HGF-MET in resistance to EGFR tyrosine kinase inhibitors in lung cancer. Curr. Signal Transduct. Ther. 6: 228233. https://doi.org/10.2174/157436211795659928

Zahreddine H, Borden K (2013) Mechanisms and insights into drug resistance in cancer. Front. Pharmacol. 4: 28. https://doi.org/10.3389/ fphar.2013.00028

Zhou Y, Kopeček J (2013) Biological rationale for the design of polymeric anti-cancer nanomedicines. J. Drug Target. 21: 1-26. https:// doi.org/10.3109/1061186X.2012.723213 